\title{
Über die Plasmorrhyse nackter Protoplasten II
}

\author{
Von H. Pfeiffer, Bremen (Deutschland)
}

Eingegangen am 29. Juni 1932

In der vorangegangenen Mitteilung haben wir die in hypertonischen Medien bewirkte Kontraktion nackter Protoplasten zum Unterschiede von dem durch H. DE VRIES aufgestellten Begriffe der Plasmolyse umhäuteter Zellen nach BaLBIANI (1, S. 537) als Plasmorrhyse bezeiehnet (9, S. 27). Die damals an pflanzlichen Beerenperikarpzellen und an menschlichen Speichelzellen angestellten Untersuchungen haben das Ziel gehabt, die plasmorrhytische Kontraktion zu charakterisieren und ihre Variationsfähigkeit ohne völlig bestimmte Umschreibung der kausalen Bedingungen, ihre Reversibilität nach Einwirkung hypotonischer Lösungen (Deplasmorrhyse) und den Einflu $B$ vorausgegangener Tanninbehandlung der Objekte zu untersuchen.

Dabei sind auch schon neue Aufgaben aufgetaucht, wie die Frage nach dem Einflu $B$ der Konzentration hyper- und hypotonischer Lösungen einerseits und der $\mathrm{C}_{H}$ der Medien andererseits (9, S. 30), an deren Beantwortung aber erst soweit herangegangen ist, als diese Fragen in die Versuche zur messenden Behandlung der Plasmorrhyse hineinreichen. Weitere Ziele, so die Untersuchung des quellenden und entquellenden Einflusses bestimmter Salzionen ( 9, S. 33), der Wirkung von Anelektrolyten und Anästhetika (womöglich bei wechselnder $\mathrm{C}_{\mathbf{H}}$ ), sind sogar noch weniger in Betracht gezogen.

Vielmehr ist bei dieser zweiten Mitteilung vor allem die Beantwortung der vorläufig wichtigeren Frage nach der quantitativen Erfassung der Erscheinung (9, S. 33) zu fördern versucht worden, und ob auch die Versuche darüber noch nicht als abgeschlossen gelten dürfen, sind doch schon einige Möglichkeiten, die einen weiteren Ausbau lohnend erscheinen lassen, anzuführen $(\$ \S 5-7)$.

Gleichzeitig ist das Bestreben darauf gerichtet gewesen, weitere Objekte der Untersuchung zugängig zu machen. Während die vorangegangenen Beobachtungen vor allem an Material aus dem saftig zerfließenden Perikarpgewebe von Beeren, also an zwar fast das ganze Jahr vorhandenen Objekten angestellt worden sind, die aber im Verlaufe der normalen Histogenese sich ihrer Membran selbst entledigen, ist nun versucht worden, pflanzliche Protoplasten experimentell ihrer Wand $z u$ entblössen ( $\$ 1)$. Daneben sind wie in dem ersten Beitrage (9, S. 31 f.) auch wieder menschliche Speichelzellen zur Untersuchung benutzt worden $(\S 7)$. 
Während nun die früher herangezogenen Objekte in der üblichen Weise bei durchfallendem Lichte mikroskopiert werden können, wobei sich dann allerdings für länger fortgesetzte Beobachtungen die Technik der Durchströmung des Präparats empfiehlt $(\$ 2)$, ist dieses Verfahren bei der Verwendung der neuen Objekte zweckmäBig durch Umstellung auf die Technik der Auflichtmikroskopie, wie sie insonderheit VoNWILLER $(14 ; 15)$ erarbeitet hat, zu ergänzen. In ihrer neueren Ausgestaltung ist diese Technik auch zur Lösung cytologischer und protoplasmatischer Probleme geeignet (12); doch bedarf es wegen ihrer bislang ziemlich seltenen Anwendung auf diesen Gebieten hier einiger kurzer Bemerkungen ( $\S 3-4)$.

\section{A. Die Beschaffung neuen Materials}

1. Die experimentelle Entblössung pflanzlicher Protoplasten gelingt am einfachsten durch Zertrümmerung der Membran ( 6 , S. 102). Oft ist auf solche Weise das dem geöffneten Lumen entschlüpfende Protoplasma, etwa bei Verwendung von Siphoneen-Zellen, in Form zahlreicher kleinerer oder größerer Tropfen gewonnen worden. Daß aber durch Öffnen plasmolysierter Zellen höherer Pflanzen auch ganze Protoplasten anscheinend ohne erhebliche Läsion zu erhalten sind, welche bei nachwirkender Einwirkung hypotonischer Lösungen aus der geöffneten Zelle herausschlüpfen, hat zuerst af KLERCKER (4, S. 465) gezeigt und ist seitdem mehrfach und zu verschiedenen Untersuchungszielen wiederholt worden.

So hat Küster (in dem Festbande für W. Roux) das Verfahren durch experimentelle Fusion benachbarter nackter Protoplasten zu einer Technik für die Darstellung doppelkerniger, abnorm großer Protoplasten ausgebaut (5, S. 1035; 6, S. 54), während W. SEIFRIz unter Anwendung mikrodissek torischer (mikrurgischer) Verfahren die nackten, plasmolysierten Protoplasten oder Tonoplasten aus ihrem Wandgehäuse herausgezogen und neuerdings R. CHAMBERS und K. HöFLER gewisse zuvor von CHAMBERS und P. REZNIKOFF vorgenommene mikrurgische Eingriffe auf die gleichen Objekte angewandt haben. Auf diese (hauptsächlich in Protoplasma Bd. 3 und 12 erschienenen) Untersuchungen in einem weiteren Beitrage dieser Serie zurückzukommen, wollen wir uns noch vorbehalten (vgl. aber auch hier schon: 3, S. $451 \mathrm{f}$.).

Die Methode af KLERCKERs (4, S. 465) besteht darin, daß Blattstücke oder, wie hier von uns vorgezogen, ganze Organe von außen her soweit plasmolysiert werden, da $B$ sich die Protoplasten allseitig von der Wand ihrer Zellen abheben und zur Kugelform zusammenziehen, worauf die Gewebe zerschnitten oder die vorbehandelten Organe mit scharfen Einschnitten versehen werden. Dann werden zwar viele der Protoplasten vernichtet werden, aber viele andere bleiben von dem Schnitte unberührt, indem er nur die Zellwandung zertrümmert. Aus 
den geöffneten Zellen kann man die infolge der plasmolytischen Vorbehandlung geschrumpften Protoplasten schon durch sanften Druck auf das Deckglas herausquetschen oder nach der von SEIFRIZ geübten Arbeitsweise herausziehen. Doch werden sie auch durch Verdünnen der vorher hypertonischen Lösung so leicht zum Ausschlüpfen gebracht, daß HUBER und HöFLER (3, S. 454, 459) nur durch besondere Maßregeln ihr Verbleiben in den geöffneten Zellen ermöglicht haben.

\section{B. Die mikroskopische Beobachtung (nebst Bemerkungen zur Technik der Auflicht-Cytoskopie)}

2. Die mikroskopische Untersuchung der nackten Protoplasten kann in der üblichen Weise im durchfallenden Lichte erfolgen, nachdem viele von ihnen mittels lose aufgedrückten Pinsels auf das Deckgläschen der Beobachtungskammer gebracht worden sind. Als solche hat sich der Durchfluss-Objektträger von E. Busch (Rathenow) mehr noch als andere im Handel befindliche Kammern bewährt. ${ }^{+)}$

Jener Apparat (Fig. 1) besteht aus einer Metallplatte von Form und Größe der üblichen Objektträger und zeigt in der Mitte einen sehr flachen zylinderischen Behälter, dessen Boden von einem wasser- und gasdicht in die Metallplatte eingelassenen Glasfenster, dessen Decke aber von einem kreisförmigen Deckglas (Durchmesser $22 \mathrm{~mm}$ ) gebildet wird, das durch einen abdichtenden Gummiring und einen mit Gewinde
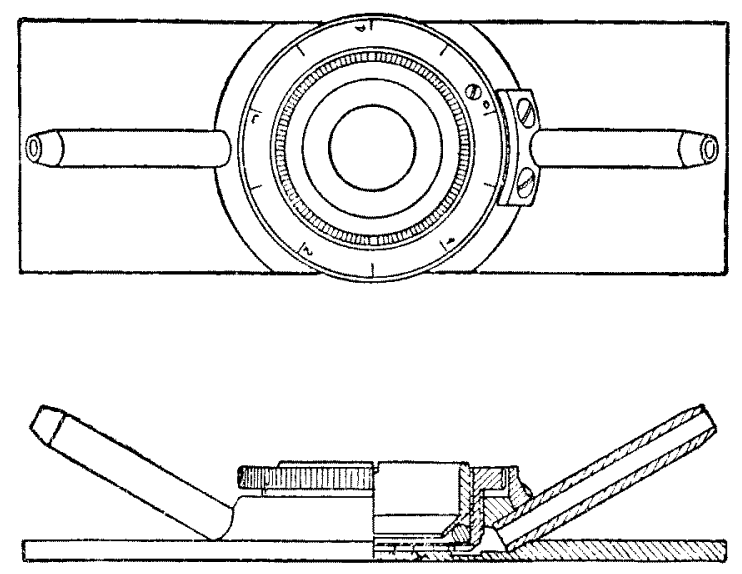

Fig. 1. Der Durchfluss-Objeltträger (E. BuscH, Rathenow) in Projektion von oben und im Querschnitt.

+) Der Firma E. Busch und insbesondere Herrn Dr. K. Albrecht möchte Verf. für die längene Überlassung einer Apparatur zu Versuchszwecken auch an dieser Stelle seinen besonderen Dank aussprechen. 
versehenen, hohlzylinderischen Deckelring festgehalten wird, welcher durch einen passenden Schlüssel heraus- oder aufschraubbar ist. In die Kammer, welche durch Drehbewegung des Deckelringes entsprechend einer angebrachten Skala auf beliebige Tiefen bis $\mathrm{zu}$ maximal $0,5 \mathrm{~mm}$ senkrechtem Abstand variiert werden kann, mündet an den Schmalseiten des Objektträgers je eine Öffnung mit Schlauchansatz. Die Maße der Kammer und die Dicke ihres Boden- und Deckglases erlauben auch bei Verwendung stärkerer Trockenobjektive oder Immersionen die erforderliche Annäherung. Überaus bequem ist nach Herausnehmen des Deckglases daran die Anbringung der zu untersuchenden Kultur im hängenden Tropfen, und auch die Reinigung der Apparatur läßt sich nach Öffnen der Kammer mühelos in der sonst üblichen Weise mittels der von ZETTNOW angegebenen Fluissigkeit vornehmen (11, S. 210 f.).

Außerdem ist in steigendem Maße auch im auffallenden Lichte mikroskopiert worden, indem gerade diese Technik mehr als die bisher gebräuchlichere die Beobachtung in vivo et in situ ermöglicht und damit die von der neueren Protoplasmaforschung erhobene Forderung nach Lebendbeobachtung erfüllt (12, S. 558). Dazu werden die unverletzten Pflanzen direkt unter das Mikroskop gebracht (Fig. 2-3), wobei die neueren Mikroskopstative auch wohl eine Zerlegung des Instruments und u. U. die Anbringung allein des Tubus mit seiner optischen Ausrüstung an einem Einfach- oder Doppeltsäulenstativ erlauben (12, S. 559).

3. Bei der Apparatur für schräg auffallendes Licht, wie sie in einer gewissen Vollkommenheit für ophthalmologische Aufgaben in der GULLSTRANDschen Einrichtung (Spaltlampe und Hornhautmikroskop nach CZAPSKI oder GREENOUGH) geschaffen worden ist und weiterhin in den Vorrichtungen des alten LIEBERKüHNspiegels (1738), des AuflichtDunkelfeldkondensors (F. HAUSER), Parabolspiegels (P. METZNER) und Schräglichtilluminators zur Verfügung steht, ergeben sich bei den in der Cytologie erforderlichen Objektiven kürzerer Brennweiten wegen des geringen Objektabstandes und der Behinderung durch die $\mathrm{Ob}$ jektivfassung technische Schwierigkeiten und schließlich Grenzen der Anwendbarkeit, die oft gerade bei den cytologisch wichtigen Vergrößerungen beginnen (15, S. 464). Das ganz besonders für diese Verhältnisse ausgebaute Verfahren vertikaler Beleuchtung von oben (15, S. 466f.) unter Ausnutzung des Objektivs gleichzeitig als Beleuchtungskondensor und Beobachtungssystem (sogen. Vertikalilluminatoren) ergibt wiederum vielfach sowohl in der Ausführung mit Planparallelglas als reflektierendes Element (BECK), als auch in jener mit total reflektierendem Prisma (NACHET) infolge mancherlei Reflexe am Tubus und an den Objektivlinsen ein teilweise verschleiertes Bild. So sind denn bei dem heutigen Stande der Auflichtmikroskopie und des Instrumentenbaues immer noch beide Verfahren nebeneinander erwünscht (12, S. 560). 
Fig. 2
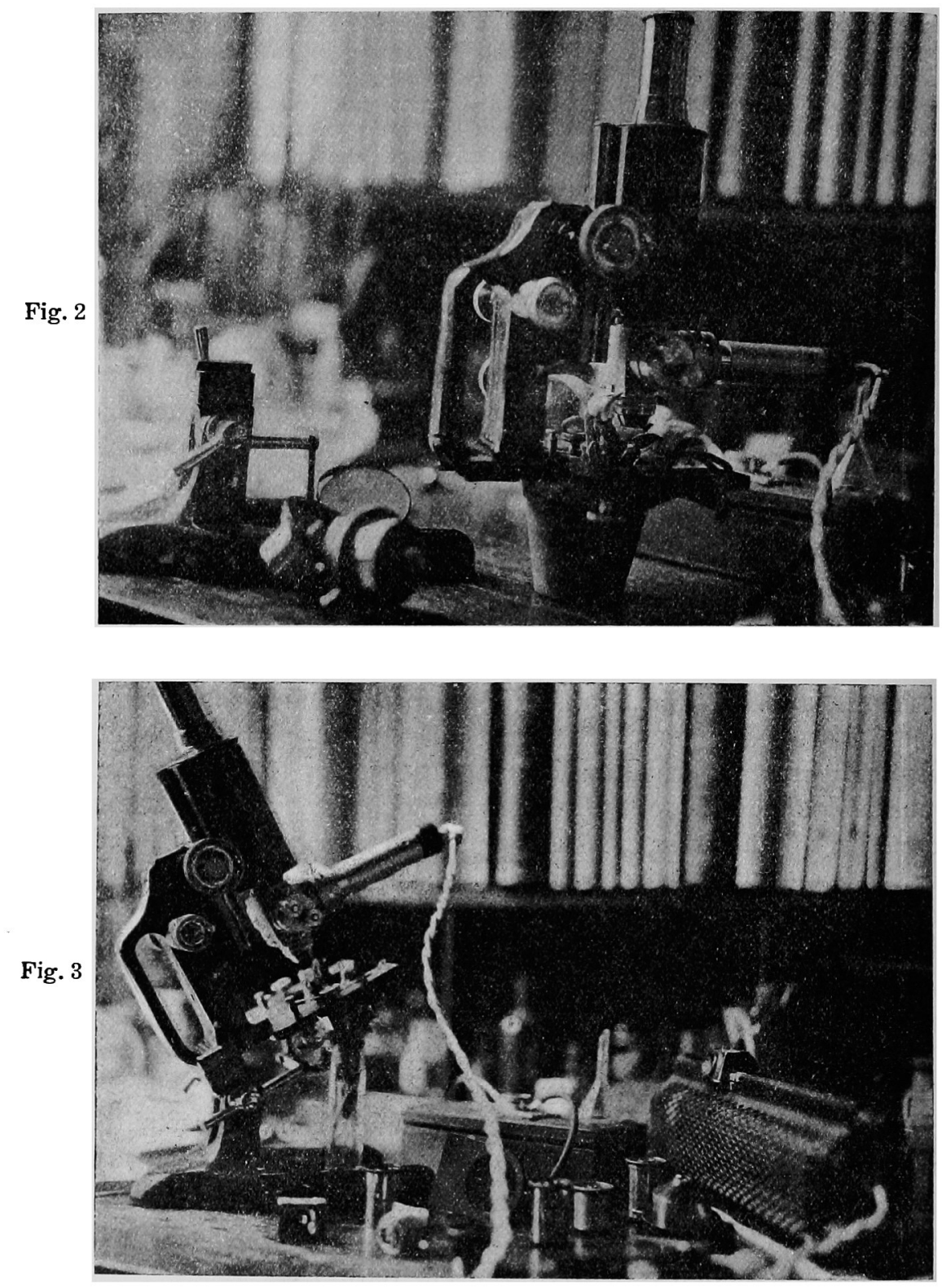

Fig. 2-3. Versuchsanordnung zu auflichtmikroskopischen Beobachtungen mit dem ,, Univertor " und einem zerlegbaren Mikroskopstativ. 
Von den beiden Reflexionselementen liefert das Prisma lichtstärkere Bilder, aber infolge des etwas verschiedenen Lichteinfalles zuweilen auch unliebsame Bildstōrungen, während das Planglas ein milderes, gleichmäßiger verteiltes Bild erzeugt, also eine zwar lichtschwächere, aber genauere Abbildung ermöglicht. Es genủgt daher bei stark reflektierenden Objekten in jeder Weise, während bei schwach reflektierenden vielfach das Prisma vorzuziehen ist. Stets aber bleibt es wïnschenswert, da $B$ beide Reflexionselemente an demselben Instrument vereinigt und auf einfachste Weise und ohne Störung der mikroskopischen Einstellung austauschbar sind (vgl. 15, S. 466).

Weitere Vervollkommnungen ergeben sich beim Spaltopakilluminator (15, S. 466) durch eine verschiebbare Konvexlinse zur Einstellung des Lichtbündels auf die Ebene des Objekts, durch gegeneinander auswechselbare Blenden zum Herausschneiden eines beliebig abzustufenden und parallel verschiebbaren Bandes aus dem Gesichtsfelde (Spaltblende) und zur ausschlieBlichen Beleuchtung eines beliebig zu verkleinernden punktförmigen Feldes (Winkelblenden).

Indem die Technik der Auflichtmikroskopie nicht die heute gebräuchlichere Anwendung durchfallenden Lichtes ersetzen, sondern nur ergänzend neben sie treten soll (15, S. $462 ; 12$, S. 559), verdient vielleicht ein universales Instrument den Vorzug, das ohne Veränderung der mikroskopischen Einstellung sämtliche Beobachtungsweisen neben- und nacheinander erlaubt. ${ }^{+}$Eine solche Möglichkeit ist in vorzüglicher Weise in dem von E. BuSCH (Rathenow) gebauten , Univertor " (10, S. $105 \mathrm{f}.)^{++)}$gegeben.

Diese Vorrichtung besitzt für die Anwendung schrägen Auflichtes (mit ausgezogenem Beleuchtungsstutzen) einen Ringspiegel im Stutzen und einen asphärischen Hohlspiegelring außerhalb des Strahlenganges des Objektivs, für die Benutzung vertikalen Auflichtes (mit eingedrücktem Stutzen) wie andere Vertikalilluminatoren die beiden auswechselbaren Reflexionselemente (Planglas und Prisma) und kann endlich zum Mikroskopieren mit durchfallendem Lichte benutzt werden, indem der Schiebestutzen eingedrückt und die Reflexionsvorrichtungen außer Tätigkeit gesetzt werden $(10$, S. 104 f.). Durch das vorhandene kleine Niedervoltglühlämpchen ist die praktische Handhabung sehr vereinfacht. Auch wird eine sehr große Reflexfreiheit bei hoher Beleuchtungsintensität erreicht und kann das Azimut von allseitiger bis zu einseitiger Beleuchtung verändert werden.

Seit kurzem liegen erste Berichte ùber einen neuen Vertikalilluminator von LEITZ (Wetzlar), den sogen. Ultropak, vor, der âhnlich wie eine von CHAPMAN und ALLDRIDGE (1924) vorgesehene Anordnung grundsätzlich das Prinzip der Beleuchtung außerhalb des Strahlenganges der Beobachtungsoptik verfolgt (vgl. die Beschreibung H. Heines in Z. wiss. Mikrosk. 49, 456, 1932). Nachdem mir von der Frima die Einrichtung zur Erprobung ihrer Eignung für cytologische Aufgaben lāngere Zeit zur Verfügung gestellt gewesen ist (wofür auch an dieser Stelle herzlicher Dank ausgedruickt sei), bemerke ich, daß die hohe Lichthelligkeit der Bilder, ihre aus dem angewandten Beleuchtungsprinzip folgende große Reflexfreiheit, sowie die Anwend-

+) Sofern auch im durchfallenden Lichte mikroskopiert werden soll, bleibt die Untersuchung allerdings auf vorbereitete Präparate statt auf den intakten Organismus beschränkt.

++ ) Für die Ermöglichung einer Erprobung durch Ưberlassung einer vollständigen Einrichtung des zerlegbaren Stativs $\mathbf{E}$ und des Univertors mit sämtlichem Zubehör bis herab zu Objektführern, Widestand u. a. Nebenapparaten (Fig. 2-3) ist Verf. der Firma und ganz besonders Herrn Dr. K. ALBRECHT zu ausserordentlichem Danke verbunden, den er hier nochmals ausdrücken möchte. 
barkeit auch der starken Immersionen U-O $75 \times \mathrm{W}$ und $90 \times \mathrm{W}$ den Ultropak für die Auflichtcytoskopie in hervorragendem Maße empfehlen, auch wenn bei ihm im Gegensatz zum Univertor eine Umstellung auf die beiden Modi der Auflichtbeleuchtung und auf die Benutzung transmittierenden Lichtes nicht möglich ist.

4. Eine ausreichende mikroskopische Auflösung der Objekte mittels auffallenden Lichtes ist, wie häufig durch Untersuchungen auf den verschiedensten Gebieten hauptsächlich von VoNWILLER und seinen Mitarbeitern V. DEMOLE, E. BoRs u.a., aber auch durch alle eigenen Beobachtungen immer wieder belegt worden ist, vor allem abhängig von geeigneter Verteilung reflektierender Strukturelemente mikroskopischer und höherer Größenordnung in dem untersuchten Objekt (12, S. 562 f.). Daß sich im gleichen Sinne aber auch die physikalische Natur der wirksamen Reflektoren (vgl. VoNWILLERs diesbezügliche Einteilung od. meinen Berliner Vortrag: 12) auswirkt, soll darum nicht übersehen werden. Die theoretische Analyse der Reflexionsverhältnisse lebender Objekte durch VoNwILLER hat uns solcherart nicht allein das Zustandekommen des Bildes verstehen, sondern auch ungünstige Reflexionsbedingungen mit Erfolg überwinden gelehrt. So brauchen wir heute die auflichtmikroskopische Untersuchung nicht einzustellen, weil ein bestimmtes Objekt nicht geeignet erscheint, sondern können die Schwierigkeiten durch Application künstlicher und geschickte Ausnützung etwa vorkommender Bioreflektoren überwinden (15, S. 464 f.).

Störungen infolge über dem Objekt befindlicher Reflektoren, die u. U. jegliche Beobachtung verhindern können, werden durch Vermeiden des Deckglases oder durch Auftragen einer sehr dünnen Lage derselben Immersionsfluissigkeit unter dasselbe, durch Drehen des Prismas oder des Planglases, durch Veränderung der Blende am Fenster des Beleuchtungsstutzens oder unter Verwendung einer besonderen Lichtquelle durch deren Äbricken, Änderung des Lichteinfalles o. dgl., beseitigt (12; vgl. auch 15 , S. 465).

Bei Schwierigkeiten infolge starker Reflexion der Aussenschicht der Protoplasten ist vielleicht nur durch Anwendung geneigten Auflichtes weiterzukommen, doch sind Hindernisse dieser Kategorie erfreulicherweise anscheinend ziemlich selten (12).

Wenn eine in geringer Tiefe im Objekt gelegene Schicht als Reflektor wirkt, so daß sich die tiefer situierten Zellen der Untersuchung entziehen, so kann man mit VONWILleR (15, S. 471 f.) die vertikale Beleuchtung durch experimentelle Abschrāgung des Lichteinfalles innerhalb der Gewebe modifizieren (indirekte Beleuchtung).

Ganz besonders bedeutsam aber wird an manchen der hier untersuchten Objekte eine andere Technik, welche ich in Anlehnung an die glänzenden Ergebnisse VoNWILLERs zusammen mit VANNOTTI in der Capillaroskopie am lebenden Menschen (16, S. 1549 f.) unabhängig von ihrer Anwendung des gleichen Prinzips zur Karyoskopie an der lebenden menschlichen Haut (17, S. $527 \mathrm{f}$.) versucht habe. Es wird dazu ein feinpoliertes, ganz ebenes oder leicht nach oben gekrümmtes $A g$ - oder Pt-Plättchen, das womöglich an der Vorderseite etwas dünner sein soll, 
möglichst flach (fast seitlich) in das Gewebe hineingeschoben. Schreitet man dann beim Mikroskopieren vom Einführungsrande proximal $a b$ wärts, so findet sich leicht die Zone, in welcher darüber situierte Schichten das spiegelnd reflektierte Licht genügend gedämpft haben.

Durch alle diese Maßnahmen glückt es, auch an sonst ungeeigneten Objekten die Vergrößerungen bis auf die cytologisch erforderlichen auszudehnen und wie sonst mikrurgische und chirurgische Eingriffe (15, S. $472 \mathrm{f} ., 473 \mathrm{f}$,) vorzunehmen.

\section{Ansätze zu quantitativer Erfassung der plasmorrhytischen Formänderungen}

5. Die in mehrfacher Hinsicht $\mathrm{zu}$ bemerkende Parallelität zwischen der Plasmolyse umhäuteter und der Plasmorrhyse nackter Protoplasten (9, S. 32 f.) legt den Gedanken nahe, für die quantitative Analyse der plasmorrhytischen Schrumpfung ähnliche Methoden zu entwickeln, wie sie in manchen Merkmalen der Plasmolyse, beispielsweise zur Messung der Viscosität durch die glückliche Pioniertätigkeit vor allem FR. WEBERs, gefunden worden sind. 'Dabei scheint freilich die verschiedene Form plasmorrhysierter Protoplasten wegen ungenügender Charakterisierbarkeit vorläufig besser auszuscheiden. Untersuchungen in dieser Richtung sind aber auch deshalb schon früh aufgegeben worden. weil die Formbestimmung auch beim Plasmolyseprozeß wegen zu geringer Empfindlichkeit der Reaktion mindestens als alleiniges Verfahren heute wohl zumeist verlassen worden ist. Dort aber hat sich, in die neuere Protoplasmaforschung wiederum durch FR. WEBER (18, S. 623) eingeführt, die Ermittlung der Plasmolyse-Zeit (vom Zutritt des Plasmolytikums bis zum Erreichen konvexer Plasmolyse) oder der Grenzplasmolyse-Zeit (bis zum Eintritt der Grenzplasmolyse) schon verschiedentlich bewährt, so zur Ermittlung des Viscositätsminimums in der Zone um den isoelektrischen Punkt (8, S. 87,88; vgl. auch 7 , S. 1584 f.) ${ }^{+1}$.

So ergibt sich das Ziel, analog der WeBERschen Methode nunmehr eine solche der Plasmorrhyse-Zeit zu entwickeln, sofern sich auf die Zeit bis zur Erreichung eines definierbaren Plasmorrhysegrades bindende Schlüsse gründen lassen.

+) Durch persönliche Mitteilung erfahre ich auf der Tagung der Dtsch. Botan. Ges. (Berlin, Pfingsten 1932) von Collegen STrugGer, daB die in dieser Weise angewandte Plasmolyse-Zeit-Methode auch schon einen wertvollen AufschluB über die antibathe Beziehung $\mathrm{z}$ wischen isoelektrischer Reaktion und Wachstumsverhalten (vgl. 7, S. 1596) geliefert hat. 
Während nun aber bei der WeBERschen Methode in dem Erreichen der konvexen oder der Grenzplasmolyse (je nach der zur Anwendung kommenden Spezialform des Verfahrens) ein fest begrenzter Zeitraum (beginnend mit dem Zutritt des Plasmolytikums) sogleich gegeben ist, fehlt ein solches Merkmal bei der Plasmorrhyse nackter Protoplasten.

Da könnte man nun zuerst die Geschwindigkeit der Volumänderungen bis zum Erreichen der vorher eigentiumlichen Dimensionen beobachten. So haben denn auch schon manche Autoren gelegentlich aus raschem und perfektem Rückgang der plasmorrhytischen Schrumpfung auf geringe Festigkeit und hohes Ausdehnungsvermögen der plasmatischen Oberfläche, aus langsamen Volumänderungen aber (wobei bei den hier erprobten Objekten zumal in angesäuertem Medium innere Plasmateile bruchsackartig hervorquellen können) auf größere Starrheit geschlossen. Es muß leider unentschieden bleiben, ob und in welcher Weise derartige Konsistenzunterschiede oberflächlicher Lagen Rückschlüsse auf das physikalische Verhalten der Gesamtprotoplasten zulassen.

Dazu möchte vielleicht die quantitative Bestimmung des Schrumpfungsgrades (in Prozenten des Ausgangsvolumens) auf mikrometrischem Wege noch besser geeignet sein. Hierzu seien einige Ergebnisse in Auswahl mitgeteilt, obgleich ein solches Verfahren durch die später mitzuteilenden Experimente noch ausgebaut werden kann ( $(6)$.

Läßt man einr 0,8 GM NaCl-Lösung auf die Außenepidermis der Zwiebelschuppe von Allium Cepa 13 Std. einwirken und nimmt dann den Schnitt vor (\$1), so ergibt die Deplasmorrhyse der Protoplasten geöffneter Zellen nach Absaugen und Auswechseln der vorherigen $\mathrm{NaCl}$-Lösung durch eine von 0,5 GM eine Größenzunahme der

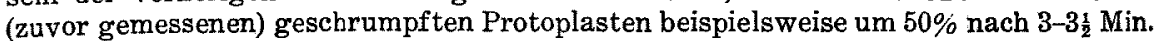
Verlängert man die Dauer der Vorbehandlung (erhöht man also wohl den Plasmolysegrad) auf vielleicht 2 (bezw. 21 ) Std., so ergibt sich die gleiche 50\%-ige Volumzunahme erst nach $6 \frac{1}{2}-7$ Min. (bezw. 7-71) Min. Durch Verwendung einer gleich starken, aber aus $\mathrm{KCl}$ und $\mathrm{CaCl}_{2}(9: 1)$ gemischten Lösung wird die Deplasmorrhyse-Zeit bis auf 3 Min. abgekürzt. Wird endlich der Scbrumpfungsrückgang der 2 Std. mit 0,8 GM jenes Gemisches vorbehandelten Objekte nach Anbringen des Schnittes mit 10\% Saccharose, welche durch Essigsäurezusatz auf eine $\mathrm{C}_{\mathbf{H}}$ von $5 \cdot 10^{-8}(\mathrm{pH} 7,5)$ oder

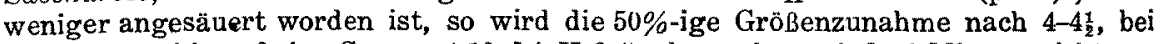
Ansäuerung bis auf eine $\mathrm{CH}_{\mathrm{H}}$ von $4 \cdot 10^{-7}(\mathrm{pH} \mathrm{6,4)} \mathrm{oder} \mathrm{mehr} \mathrm{nach} \mathrm{8-10} \mathrm{Min.} \mathrm{erreicht.}$

In Versuchen mit den an der Pflanze belassenen Blättern von Convallaria majalis, bei denen nach Plasmolyse mittels $0,8 \mathrm{GM}$ Fructose nachher in 0,6 (bezw. 0,5 ) GM deplasmorrhysiert worden ist, zeigt sich eine viel schnellere Volumsteigerung, sodaB eine $75 \%$-ige Zunahme gegenüber dem Volumen des geschrumpften Protoplasten als Grenzwert angenommen werden muB, der je nach Alter der Blätter und ihrer Zellen oder

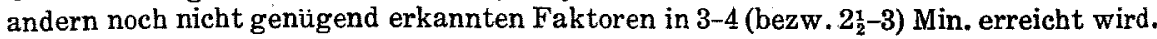

Bei andern Objekten und wechselnden osmotischen Medien werden wir uns also zweckmäßig nicht an die Bestimmung der 50\%-igen Volumzunahme bei der Deplasmorrhyse binden dürfen. Die geringeren Schwellungsgrade eignen sich allerdings sowohl wegen ihrer schwierigeren Bestimmbarkeit, als auch wegen bisweilen zu findender Unregelmäßigkeiten in der deplasmorrhytischen Schwellung wenig oder garnicht als Maßsstab für die Formänderungen. 
6. Gleicht nun die eben charakterisierte Methode der Bestimmung der Deplasmorrhyse-Zeit durchaus der von HUBER und HöFLER ( $3, \mathrm{~S}$. 370) angegebenen Ermittlung der ,Halbwertszeit" in der Deplasmolyse, in welcher die Hälfte der Endverkleinerung erreicht oder ein anderer fixer ,, Diminutionsgrad “ (3, S. 369) verglichen wird, so muß das Verfahren durch Annahme der plasmometrischen Technik HöfLERs (1918) auch in dem von jenen Autoren dargelegten Sinne zur kurvenmässigen Bestimmung der Plasmorrhysegrad-Zeit-Werte geeignet sein. $\mathrm{DaB}$ diese Erwartung voll berechtigt ist, beweisen ja schon gewisse ihrer Versuche mit Epidermiszellen von Allium (Außenlage der Zwiebelschuppen), in denen sie allerdings andere Ziele (Ermittlung von Permeationskonstanten) verfolgen und das Ausschlüpfen der Protoplasten wegen des Vergleiches jener in geschlossenen und in geöffneten Zellen möglichst auszuschließen trachten (

Unter der Voraussetzung, daß die Geschwindigkeit der deplasmorrhytischen Volumzunahme der Protoplasten in jedem Augenblick dem osmotischen Gradienten von der Außenlösung zum Plasmainnern proportional ist, werden wir also nach Ermittlung der Dimensionen des aus dem Zellwandgehäuse befreiten Protoplasten nur für möglichst viele Zeitpunkte die Dimensionsänderungen bestimmen, um daraus für jene Zeiten das relative Volumen des deplasmorrhysierten Körpers im Vergleich zu dem bei Versuchsbeginn zu finden (s. die Tab. u. Fig. 4).

Welche Vorsichtsmaßnahmen zur Vermeidung von Fehlmessungen ùber die tunlichste Konstanthaltung von Temperatur, chemischen, osmotischen, Aciditätsbedingungen usw. hinaus angewendet werden sollten, lehrt die Plasmometrie. Doch sind manche der Vorschriften bei auflichtmikroskopischer Untersuchung nicht gut durchführbar. Beispielsweise werden Verzögerungen in der Deplasmorrhyse kaum in völlig befriedigendem Ausmaße ausgeschlossen werden können. Daß im übrigen die Bestimmung der Deplasmorrhysegeschwindigkeit, ob auch auf andere Permeationskonstanten als jene des plasmolytischen Schrumpfungsgrades basiert, vollauf berechtigt ist (praktisch sogar vielfach den Vorzug verdient), ist durch die reichen Erfahrungen HöFLERs und seiner Mitarbeiter sicher erwiesen (vgl. 3, S. 470 f.; s. aber auch S. 480). Ihm verdanken wir auch eine mustergilltige mathematische Behandlung des Versuchsmaterials (3, S. 361 f.), die hier daher nicht erst gebracht zu werden braucht.

Die vorausgesetzte einfache Beziehung der Volumänderung zum Konzentrationsgefälle gilt u.a. nur unter der Bedingung, da B sich das osmotisch wirksame Material der Zelle unverändert erhält und nur proportional des Volumrückganges concentriert, nicht aber eine Hemmung oder Beschleunigung durch Ana-oder Katatonose eintritt. Anscheinend liegen freilich Störungen dieser Art, die mir theoretisch durchaus möglich erscheinen, weit unter der erreichbaren Fehlergrenze. Vorausgesetzt wird ferner das Ausbleiben von Endosmosen und Exosmosen; wesentliche Störungen in dieser Hinsicht sind nach den gegenwärtigen Erfahrungen auch bei den hier untersuchten Landpflanzen kaum zu erwarten.

Eine zuweilen erhebliche Unübersichtlichkeit erfahren die Messungsbefunde durch individuelle Verschiedenheiten der Protoplasten nicht allein nach ihrer Größe, sondern auch hinsichtlich ihres osmotischen und Quellungsverhaltens. Wie für andere indivi- 
duelle Differenzen dürfte auch hier die von A. PÁAL entwickelte Beziehung Geltung haben, nach welcher unter optimalen Bedingungen die Variationen ein Minimum erreichen, mit der Verschlechterung der Untersuchungsbedingungen aber entsprechend anwachsen.

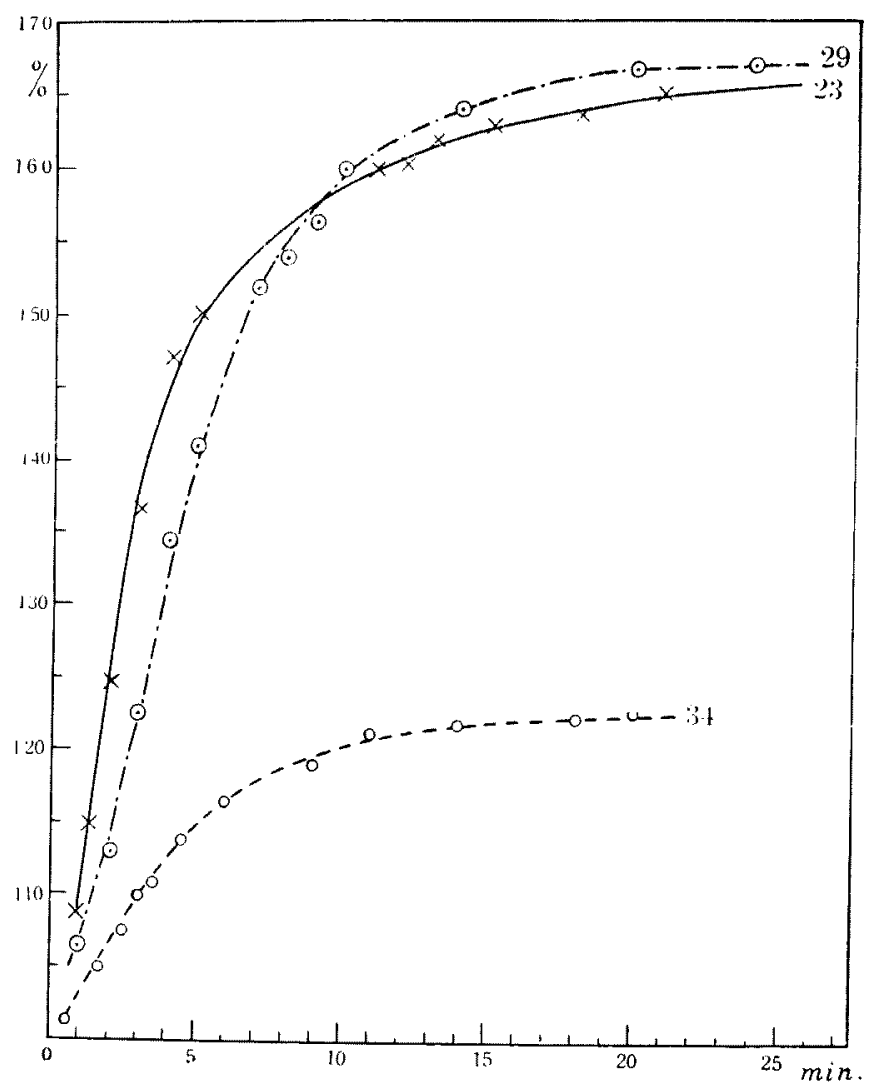

Fig.4. Verlauf der deplasmorrhytischen Schwellung nackter Allium-Protoplasten: Vers. $23(\times-\times)$ in reiner NaCl-Lösung; Vers. $29(\odot-\cdot-\cdot-\odot)$ in dem erwähnten Gemisch aus $\mathrm{KCl}$ und $\mathrm{CaCl}_{2}$; Vers. $34(\mathrm{O} \ldots \mathrm{O})$ in Saccharose.

Trotz mancher heute noch nicht vermeidbarer Übelstände dürfte die Methode der ihr gestellten Aufgabe (s. Einleitung) gerecht werden, wie Versuche mit Lösungen verschiedenen Concentrationsgrades zeigen (vgl. auch Fig. 4). Anscheinend ist bei diesen und andern Unterschieden das Eingreifen von Quellungsphänomenen in hervorragendem Maße beteiligt, wie nach älteren Angaben von W. PFEFFER (1897) und neueren von H. Schade auf klinischem, von H. KotTe und vor allem H. WALTER 
auf botanischem Gebiete auch zu erwarten ist. Da die Versuche aber noch fortgesetzt werden, mögen die Folgerungen über den Einfluß bestimmter Salzionen (s. Einleitung) auf die Erscheinung vorläufig noch zurückgestellt werden.

\section{Tabelle}

der prozentualen Deplasmorrbyseschwellung von Allium-Protoplasten im Vergleich zu dem Volumen (100\%) plasmolytischer Schrumpfung (vgl. Fig. 4).

\begin{tabular}{|c|c|c|c|c|c|c|c|c|c|c|c|c|c|c|c|c|}
\hline \multirow{2}{*}{$\mathrm{Nr}$} & \multicolumn{4}{|c|}{$\begin{array}{l}\text { Versuchs- } \\
\text { Modus : }\end{array}$} & \multicolumn{12}{|c|}{ Zeit nach einsetzender Deplasmorrhyse in Min. } \\
\hline & \begin{tabular}{|c|} 
Daner der \\
Vorbe- \\
handung \\
in Std.
\end{tabular} & $\mathrm{C}_{1}^{\prime}$ & $\begin{array}{l}\text { Me- } \\
\text { dium }\end{array}$ & $C_{2}$ & $\frac{1}{2}$ & 1 & $1_{2}^{1}$ & 2 & $2 \frac{1}{2}$ & 3 & $3 \frac{1}{2}$ & 4 & $4 \frac{1}{2}$ & 5 & 6 & 7 \\
\hline 23 & 2 & $\begin{array}{l}0,8 \\
\text { GM }\end{array}$ & $\mathrm{NaCl}$ & $\begin{array}{l}0,5 \\
\text { GM }\end{array}$ & - & 108 & 115 & 124,5 & - & 136,5 & - & 147 & - & 150 & - & - \\
\hline 29 & 5 & $\begin{array}{l}0,8 \\
\text { GM }\end{array}$ & $\begin{array}{l}\mathrm{KCl}+ \\
\mathrm{CaCl}\end{array}$ & $\begin{array}{l}0,5 \\
\text { GM }\end{array}$ & - & 106,5 & - & 113 & - & 122,5 & - & 134,5 & - & 141 & - & 152 \\
\hline 34 & $1 \frac{1}{2}$ & $\begin{array}{l}1,2 \\
\mathrm{GM}\end{array}$ & $\begin{array}{l}\text { Sac- } \\
\text { cha- } \\
\text { rose }\end{array}$ & $\left|\begin{array}{l}0,95 \\
G M\end{array}\right|$ & 101,5 & - & 105 & - & 107,5 & 110 & 111 & - & 114 & - & 116,5 & - \\
\hline
\end{tabular}

\begin{tabular}{|c|c|c|c|c|c|c|c|c|c|c|c|c|c|c|c|c|}
\hline \multirow{2}{*}{$\mathrm{Nr}$} & \multicolumn{4}{|c|}{$\begin{array}{l}\text { Versuchs- } \\
\text { Modus : }\end{array}$} & \multicolumn{12}{|c|}{ Zeit nach einsetzender Deplasmorrhyse in Min. } \\
\hline & $\begin{array}{l}\text { Dauer der } \\
\text { Vorbe- } \\
\text { handlung } \\
\text { in Std. }\end{array}$ & $C_{1}^{\prime}$ & $\begin{array}{l}\text { Me- } \\
\text { dium }\end{array}$ & $\mathrm{C}_{2}$ & 8 & 9 & 10 & 11 & 12 & 13 & 14 & 15 & 18 & 20 & 21 & 24 \\
\hline 23 & 2 & $\begin{array}{l}0,8 \\
\text { GM }\end{array}$ & $\mathrm{NaCl}$ & $\begin{array}{l}0,5 \\
\mathrm{GM}\end{array} \mid$ & - & -- & - & 160 & 160,5 & 162 & - & 163 & 163,5 & - & 165 & - \\
\hline 29 & 5 & $\begin{array}{l}0,8 \\
\text { GM }\end{array}$ & $\begin{array}{l}\mathrm{KCl}+ \\
\mathrm{CaCl}{ }_{2}\end{array}$ & $\begin{array}{l}0,5 \\
\text { GM }\end{array}$ & 154 & 156,5 & 160 & - & - & - & 164 & - & - & 167 & - & 167 \\
\hline 34 & $1 \frac{1}{2}$ & $\begin{array}{l}1,2 \\
\text { GM }\end{array}$ & $\begin{array}{l}\text { Sac- } \\
\text { cha- } \\
\text { rose }\end{array}$ & $\begin{array}{l}0,96 \\
\mathrm{GM}\end{array}$ & - & 118,5 & - & 121 & - & - & 121,5 & - & 122 & 122,5 & - & - \\
\hline
\end{tabular}

7. Wenngleich es nach allen unsern Erfahrungen der Plasmometrie berechtigt ist $(\$ 6)$, statt der Plasmorrhyse den umgekehrten Prozeß der Größenzunahme der nackten Protoplasten vergleichend und messend $\mathrm{zu}$ untersuchen, so haben sich die Bemühungen doch auch darauf gerichtet, die plasmorrhytische Schrumpfung ebenfalls vergleichender Analyse näherzuführen. Indem dabei die BRownsche Bewegung $(B B)$ als Indicator Verwendung gefunden hat, schlieBen sich die Versuche an die früheren Beobachtungen an menschlichen Speichelzellen (9, S. $31 \mathrm{f}$.$) an.$ 
Wenn die in der vorangegangenen Mitteilung $(9, \mathrm{~S} .31)$ und auch von SCHADE und WeILER (13, S. 46 f.) vertretene Anschauung, daß die $\mathrm{BB}$ von einer $\mathrm{H}-\mathrm{Hyp}$ erionie oder von osmotischer Hypotonie abhängt, richtig ist, so könnte die Sistierung $\operatorname{der} B B$ als Folge plasmorrhytischer Hypertonie unter Berücksichtigung des zeitlichen Eintrittes oder der Geschwindigkeit der Reaktion ein angenähertes $\mathrm{MaB}$ ergeben. Das hat sich in der Tat gezeigt. Es genügt, dem Präparat Leitungswasser zuzusetzen, um die BB hervorzurufen und dann nach Absaugen des Mediums schnell die Salzlösung zutreten zu lassen. Je nach der Concentration des hypertonischen Mediums ist alsdann eine frühere oder spätere Einstellung der $\mathrm{BB}$ zu bemerken. So schwierig die morphologischen Verhältnisse ohne sehr langwierige Übung und Erfahrung auch $\mathrm{zu}$ vergleichen sind, so laufen doch offenbar plasmorrhytische Kontraktion und Sistierung der BB völlig symbath.

Wenn Schade (13, S. 54 f.) und PFeiffer (9, S. 28) bei entsprechender Ansäuerung einer stark hypertonischen Tyrodelösung $(\Delta 0,87)$ mit Acid. uricum puriss. $\left(\mathrm{C}_{\mathrm{H}} 2,5 \cdot 10^{-2}\right)$ eine beträchtliche Quellung und gleichzeitig BB feststellen, so ergibt sich aus der vollkommenen Übereinstimmung mit den Befunden bei osmotischer Hypotonie gleichfalls die Berechtigung für die Annahme, daßs in die untersuchten Prozesse Quellungsphänomene hineinragen $(\$ 6)$. Die recht beträchtliche $\mathrm{H}-\mathrm{Hy}-$ perionie schließt in solchen Fällen die Plasmorrhyse trotz Hypertonie des Mediums aus (deplasmorrhytischer Säureeinfluss).

Wir dürfen hier nicht unterlassen, auf eine noch ungeklärt gebliebene Unstim migkeit gegenüber Befunden VAN HERWERDENS hinzuweisen, nach denen Frosch: Leukocyten bei Übertragen in $0,1 \%$ Essigsäure (in $0,9 \% \mathrm{NaCl}$ ) ihre amöboide Fortbewegung und die BB einstellen, während beim Auswaschen mit der reinen NaCl-Lösung beide erneut einsetzen.

Wie es bei der Heranziehung der BB gewöhnlich geschieht, ${ }^{+)}$so begnügen wir uns mit der Angabe der Lebhaftigkeit oder Trägheit, Beschleunigung oder Verzögerung und Einleitung oder Beendigung der Bewegung. Die wenigen wirklich messenden Verfahren haben aber die Kenntnis des Partikelradius zur Voraussetzung und sind daher in

+) Den zahlreichen auf ähnliche Schätzungen basierten Angaben-zur Untersuchung des Viscositätsgrades beispielsweise aus neuerer Zeit jene von P. Russo, M. J. Hogue, R. Gross, E. Leblond, W. M. Bayliss, W. W. Lepeschkin, J. Chifflot und C. Gautier, W. Seifriz, F. J. Brinley u.v.a.-stehen an Messungen neben den Versuchen von L. G. M. Becking HaAs, H. v. D. Sande Bakhuyszen und H. Hottelling eigentlich nur noch die ubberaus wichtigen Untersuchungen von J. PEKAREK gegenüber, der unter Anwendung der R. FüRTHschen Methode der mittleren, doppelseitigen Erstpassagezeiten der in BB befindlichen Teilchen die absolute Viscosität auch von Protoplasten gemessen hat. 
dieser Form auf unser Objekt nicht übertagbar. Wenn wir freilich für die Viscosität des Plasmas einen Wert als bekannt einsetzen dürften, so könnte die in der letzten Note erwähnte Methode von R. FürTH bei Benutzung der Diffusionskammer nach FÜRTH und E. UlLMANN (2, S. 820) und des Diffusionsmikroskops nach J. GICKLHORN und A. Nistler (2, S. 825 f.) recht wohl zur Ermittlung der Dimension der schwingenden Teilchen benutzt werden (2, S. 816 f.), doch ist nicht ersichtlich, welchen Vorteil ein solches Ergebnis für die hier angestellten Untersuchungen haben sollte. Aus den angegebenen Gründen sind auch schon andere Versuche auf ihre Ausbaufähigkeit zu Messungszwecken bei der Plasmorrhyse untersucht worden. Indem hier aber noch keine befriedigenden Resultate erlangt worden sind, mögen ein paar kurze Andeutungen über diese noch weiter zu verfolgenden Erscheinungen die vorliegende Mitteilung beschließen.

Wie man eine Volumzunahme von Protoplasten auch wohl als eine \pm direkte Wirkung erniedrigter Oberflächenspannung der plasmatischen Außenfläche betrachten kann, so möchte die plasmorrhytische Kontraktion zugleich als Folge erhöhter Oberflächenspannung zu deuten sein. So läßt sich durch UUberführen der Speichelzellen in $1 \%$ Saponin. pur. albiss. (stalagmometrische Tropfenzahl 59) eine anfangs (2 Std.) merkbare, dann (nach 4, 8, 24 Std.) um je rund die Hälfte der jeweils vorherigen Steigerung zurückgehende Volumzunabme, in so geringen Grenzen sie sich auch hält, erkennen, während 1\% Natriumglykocholat (Tropfenzahl 95) zu diesem Versuche ungeeignet ist. Ob aber auch G. LEPEHNE (Verhdl. Dtsch. Ges. inn. Med.-Wiesbaden 1922) bei Funktionsstörungen der Leber durch Messung der Oberflächenspannung mittels der HAYKRAFT-Reaktion (7, S. 1583) klinisch brauchbare Vergleichswerte für den Gallensäurengehalt der Sera zu ermitteln vermochte, so ist doch bekannt, da $B$ für gallensaure Salze und Saponine die Wirkung auf die Oberflächenspannung und auf physiologische Erscheinungen nicht immer proportional sind (F. BOAS, L. KOFLER, u.a.). Daher sind die Versuche zur Entwicklung eines messenden Verfahrens für die Deplasmorrhyse in dieser Richtung vorläufig aufgegeben worden.

Ausgezeichnete Aussichten scheinen aber für Bestimmungen der Loslösungszeit (nach vorheriger gleicher Anheftungszeit) und für messende Versuche der Klebrigkeit (stickiness) und des Haftvermögens (adhesiveness) an Fremdkörpern unter Heranziehung etwa der Abwaschmethode von W. BARIKINE oder des Strömungsverfahrens nach W. O. FENN zu bestehen. Indem diese Methoden aber noch der näheren Ausarbeitung und der besseren Anpassung an die von uns verfolgten Ziele bedurfen, sei eine ausführliche Darstellung der Ergebnisse mit ihnen einstweilen zuruickgestellt.

\section{Zusammenfassung}

Die Fortsetzung der Plasmorrhyse- und Deplasmorrhyseuntersuchungen ist weiterhin teilweise an den schon früher berangezogenen menschlichen Speichelzellen, daneben aber an experimentell nach der Methode af KLERCKERs entblößten Protoplasten pflanzlicher Abkunft (Zwiebelschuppen von Allium, Blätter von Arten von Allium, Convallaria, Iris, Orchis. Agave und Sedum) vorgenommen worden. Die mikroskopischen Beobachtungen sind bei den Speichelzellen allein, bei den pflanzlichen Objekten teilweise im durchfallenden Lichte (unter Verwendung eines als Durchströmungskammer dienenden Durchfluß-Objektträgers) geschehen und in den letzteren 
Fällen durch ständiges auflichtmikroskopisches Arbeiten mit dem neuen llluminator ", Univertor ' (BUSCH) ergänzt worden. AuBer den Bemerkungen zur af KLERCKERschen Methode und zu den Einrichtungen zur Durchströmung des Präparats und zur Auflichtcy toskopie, die eine Untersuchung der Objekte in vivo et in situ ermöglichen soll, bringen die einführenden Kapitel Betrachtungen zum Ausbau der auflichtmikroskopischen Technik in der Cytologie (entscheidender Wert der natürlichen und Bioreflehtoren der Objekte).

Im zweiten Teile werden dann vor allem Versuche besprochen, die eine quantitative Erfassung der Plasmorrhyse und Deplasmorrhyse anbahnen helfen sollen. Wegen der Verwand tschaft der Plasmorrhyse nackter mit der Plasmolyse umhäuteter Protoplasten wird zuerst in Anlehnung an ein Verfahren FR. WEBERs ein solches zur Verknüpfung von Plasmorrhysegrad (in Prozent) mit Einwirkungszeit des Mediums versucht. Die Ähnlichkeit dieser Methode mit plasmometrischen Bestimmungen der "Halbwertszeit" durch K. HöFLER führt dann zur Erprobung mikrometrischer Ermittlungen der Volumänderungen gegen die Zeit (in Min.). Erstreben diese beiden Versuchsreiben (an den botanischen Objekten) ein quantitatives Eindringen in die Deplasmorrhyse (wobei freilich zugleich die Erfassung plasmorrhytischer Formänderungen zum letzten Ziel gesetzt worden ist), so sind die an den Speichelzellen angestellten Versuche, die $B B$ nach ihrer Lebhaftigkeit oder Trägheit, Beschleunigung oder Verzögerung und Einleitung oder Beendigung als Indicator plasmatischer Zustandsänderungen zu verwenden, auf die Analyse der plasmorrhytischen Schrumpfung selbst gerichtet. Außer über den Stand dieser Bemùhungen werden schließlich Erfahrungen mit einigen erst kürzlich begonnenen Versuchen ùber Methoden mitgeteilt, die wegen ihrer teilweise erfreulichen Aussichten noch weiter zu verfolgen bleiben.

\section{Literatur}

1. Balbiani, E. G., Etudes sur l'action des sels sur les Infusoires, Arch. Anat. micr. 2: 518 -600 (1898, ersch. 1899).

2. Fürth, R., Methoden zur Bestimmung der elektrischen Struktur kolloider Stoffe insbesondere der Biokolloide. In : E. Abderhalden, Handb. biol. Arbeitsmeth., (III), B : 775-857 (1929).

3. Huber, Br. und Höfler, K., Die Wasserpermeabilität des Protoplasmas, Jahrb. wiss. Bot. $73: 351-511$ (1930).

4. Klercker, J. af, Eine Methode zur Isolierung lebender Protoplasten, Öfvers. Vet.Akad. Förhdl. 1892 : 463-474.

5. Küster, E., Experimentelle Physiologie der Pflanzenzelle. In : E. Abderhalden, Handb. biol. Arbeitsmeth., (XI), $1: 961-1058$ (1924).

6. - Pathologie der Pflanzenzelle I. (Protopl. Monogr. 3.) Berlin (Gebr. Borntraeger) 1929.

7. Pfeiffer, H., Der isoelektrische Punkt (IEP) von Protoplasten und seine Ermittlung. In : E. Abderhalden, Handb. biol. Arbeitsmeth., (V), 2/2; 1563-1596 (1929).

8. - Kleine Beiträge zur Bestimmung des IEP von Protoplasten III, Protoplasma $14: 83-90$ (1931).

9. - U̇ber die Plasmorrhyse nackter Protoplasten in hypertonischen Medien I, Cytologia 3 : 26-35 (1931).

10. - Der neue Universal-Vertikalilluminator , Univertor “ der E. Busch A.-G., Z. wiss. Mikrosk. 49: 103-107 (1982).

11. - Zur Technik der $\mathrm{CO}_{2}$-Umströmung lebender Zellen und Gewebe, ibid. 49: 208216 (1932).

12. - Die Plasmo- und Karyoskopie im auffallenden Lichte in ihrer Bedeutung fur die botanische Cytologie und Protoplasmaforschung, Verhdl. Dtsch. Bot. Ges.-Berlin 1932, ausfuhrlich in : Protoplasma 17 : 558-570 (1933). 
13. Schade, H., und Weiler, L., Beiträge zur Kenntnis des Protoplasmaverhaltens menschlicher Zellen bei physiko-chemischer Beeinflussung, Protoplasma 3: 43-67 (1928).

14. Vonwiller, P., Histologische Methoden und Ergebnisse der Mikroskopie im auffallenden Lichte. In: E. Abderhalden, Handb. biol. Arbeitsmeth., (V), 2 : 1033-1054 (1927).

15. - Lebenduntersuchungen im auffallenden Lichte. In: T. Péterfi, Meth. wiss. Biol. 1 : 460-474 (1928).

16. - und Vannotti, A., Die Capillaroskopie mit starken Vergrößerungen. In: E. Abderhalden, Handb. biol. Arbeitsmeth., (V), 2/2 : 1529-1562 (1929).

17. - - Neue Wege der Gewebelehre IV, Z. Anat. u. Entw.gesch. 95: 512-630 (1931).

18. Weber, Fr., Plasmolyse-Zeit-Methode, Protoplasma 5: 622-624 (1929). 